\title{
Complexity of Homogeneous Co-Boolean Constraint Satisfaction Problems
}

\author{
Florian Richoux \\ florian.richoux@polytechnique.edu \\ Department of Mathematical Informatics, University of Tokyo
}

\begin{abstract}
Constraint Satisfaction Problems (CSP) constitute a convenient way to capture many combinatorial problems. The general CSP is known to be NP-complete, but its complexity depends on a template, usually a set of relations, upon which they are constructed. Following this template, there exist tractable and intractable instances of CSPs. It has been proved that for each CSP problem over a given set of relations there exists a corresponding CSP problem over graphs of unary functions belonging to the same complexity class. In this short note we show a dichotomy theorem for every finite domain $D$ of CSP built upon graphs of homogeneous co-Boolean functions, i.e., unary functions sharing the Boolean range $\{0,1\} \subseteq D$.
\end{abstract}

\section{Introduction}

Constraint Satisfaction Problems (CSP) constitute a convenient and uniform framework to describe many algorithmic and combinatorial problems from graph theory, artificial intelligence, optimization, computational molecular biology, etc. The general CSP problem is well-known to be NP-complete. However, we can consider the parametric version of the CSP problem, denoted $\operatorname{CSP}(S)$, where the template $S$ is a set of allowed relations upon which any instance of the problem is constructed. The goal is to study the complexity of the parametric CSP, recognizing the conditions allowing us to distinguish between tractable and intractable instances of the considered problem, as well as the understanding of the complexity classes to which these instances belong. The study of computational complexity of constraint satisfaction problems was started by Schaefer in his landmark paper [7, where he completely characterized the complexity of Boolean CSP, distinguishing between polynomial and NP-complete instances. Feder and Vardi 4 extended this study to constraint satisfaction problems over finite domains, for which they conjectured the existence of a Dichotomy Theorem. So far, this claim was proved only for the ternary domain by Bulatov [2], exhibiting an involved Dichotomy Theorem, whereas the claim remains open for higher cardinality domains.

A fundamental result from Feder, Madelaine and Stewart [3] shows that for every set of relations $S$, there exists a set $F$ of unary functions, such that the problems $\operatorname{CSP}(S)$ and $\operatorname{CSP}\left(F^{\bullet}\right)$ are polynomial-time equivalent, where $F^{\bullet}$ is the set of the graphs of functions from $F$. Thus, CSPs over unary functions are as powerful as general CSP problems. Graphs of unary functions give us a very structural template which is really convenient to work with. 
In this paper, we focus on templates built upon homogeneous co-Boolean functions on a domain $D$, that is, unary functions sharing a range of size two. By convention, we take the range $\{0,1\} \subseteq D$. The goal of this paper is more to present well-known results from another angle and initiate a new way to study the complexity of $\operatorname{CSP}(S)$ problems rather than to present new polynomialtime algorithms for CSP. The paper is organized as follows. The first section describes general notions used in this paper. Then we introduce the parametric CSP problem in general and more specifically on graphs of homogeneous coBoolean functions, as well as some intermediary results. In the last section, we show a dichotomy theorem for every finite domain $D$ of CSP built upon graphs of homogeneous co-Boolean functions. The paper terminates with some concluding remarks.

\section{Preliminaries}

Let $f: D \rightarrow D$ be a unary function over a finite domain $D=\{0, \ldots, n-1\}$. This function $f$ is called co-Boolean if the range of $f$, also named the co-domain, is of size 2. In this paper, we focus on homogeneous co-Boolean functions, i.e. co-Boolean functions sharing the same co-domain. By convention, we choose $\{0,1\} \subseteq D$ to be this shared co-domain. Since in this short note we deal with homogeneous co-Boolean functions only, we can simply named these functions "co-Boolean functions" without any confusions. The idea behind co-Boolean functions is a partition of the domain $D$ into two disjoint sub-domains, where $f$ acts as a characteristic function.

Since we study in this paper only unary functions, each function will be considered to be unary even if we do not explicitly mention its arity. We assume that the domain $D$ is ordered by an arbitrary but fixed total order $<$. Without loss of generality, we can assume that $<$ is the natural order $0<1<\cdots<n-1$. In other words, the algebraic structure $(D ;<)$ is a chain.

The graph of a function $f$ is the binary relation $f^{\bullet}=\{(x, f(x)) \mid x \in D\}$, extended by overloading to a set of functions $F$ as $F^{\bullet}=\left\{f^{\bullet} \mid f \in F\right\}$.

An $\ell$-ary relation $R$ on a domain $D$ is a subset of $D^{\ell}$. A relation $R$ is called 0 -valid (1-valid) if it contains the all-zeros tuple $(0 \cdots 0)$ (all-ones tuple $(1 \cdots 1)$ ). Given a tuple $t$ in a $\ell$-ary relation $R$, we denote by $t[i]$ the $i$-th coordinate of $t$, with $1 \leq i \leq \ell$. We say that a relation $R$ is closed under (or preserved by) a $k$-ary operation $p$, or that $p$ is a polymorphism of $R$, if for any choice of not necessarily distinct $k$ tuples $t_{1}, \ldots, t_{k} \in R$ the following membership condition holds:

$$
\left(p\left(t_{1}[1], \ldots, t_{k}[1]\right), p\left(t_{1}[2], \ldots, t_{k}[2]\right), \ldots, p\left(t_{1}[\ell], \ldots, t_{k}[\ell]\right)\right) \in R,
$$

i.e., that the new tuple constructed coordinate-wise from $t_{1}, \ldots, t_{k}$ by means of $p$ belongs to $R$. We denote by $\operatorname{Pol} R$ the set of polymorphisms of a relation $R$ and by Pol $S$ the polymorphisms of every relation $R$ in $S$. Recall that $\operatorname{Pol} S=$ $\bigcap_{R \in S} \operatorname{Pol} R$. 
In particular, we need to study the closure under four operations, namely majority, minority, maximum, and minimum. Maximum and minimum are binary operations, satisfying respectively the following conditions for all elements $a, b \in D$ :

$$
\max (a, b)=\left\{\begin{array}{ll}
a & \text { if } a \geq b \\
b & \text { otherwise }
\end{array}, \quad \min (a, b)= \begin{cases}a & \text { if } a \leq b \\
b & \text { otherwise }\end{cases}\right.
$$

Both aforementioned operations are known in universal algebra as semi-lattice operations, since they correspond to the operations of join and meet. On the Boolean domain $\{0,1\}$, the maximum operation $\max (x, y)$ translates to disjunction $x \vee y$ and the minumum operation $\min (x, y)$ translates to conjunction $x \wedge y$. More generally, a semi-lattice operation is a binary associative, commutative and idempotent operation. We say that a $k$-ary operation $q: D^{k} \rightarrow D$ is idempotent if the identity $q(a, \ldots, a)=a$ holds for every $a \in D$.

Majority and minority are ternary operations satisfying respectively the following conditions for all elements $a, b \in D$ :

$$
\begin{aligned}
& \operatorname{major}(a, a, b)=\operatorname{major}(a, b, a)=\operatorname{major}(b, a, a)=a, \\
& \operatorname{minor}(a, a, b)=\operatorname{minor}(a, b, a)=\operatorname{minor}(b, a, a)=b .
\end{aligned}
$$

It is clear that there can be several majority and minority operation on domains $D$ of cardinality $|D|>2$, whereas there is only one majority and one minority on the Boolean domain $\{0,1\}$. The majority and minority operations on the Boolean domain can be also expressed as major $(x, y, z)=(x \vee y) \wedge(y \vee$ $z) \wedge(z \vee x)=(x \wedge y) \vee(y \wedge z) \vee(z \wedge x)$ and $\operatorname{minor}(x, y, z)=x+y+z(\bmod 2)$

There exists a pointwise partial order $\preceq$ on any $k$-ary relation $R \subseteq D^{k}$ induced by the total order $<$ on the domain $D$ defined as follows. Two tuples $t$ and $t^{\prime}$ from a $k$-ary relation $R$ satisfy $t \preceq t^{\prime}$ if $t[i]<t^{\prime}[i]$ holds for each $i \in\{1, \ldots, k\}$. We write $t \prec t^{\prime}$ if $t \preceq t^{\prime}$ and $t \neq t^{\prime}$.

A constraint language is a set $S$ of relations over the domain $D$. Let $X$ be a finite set of variables. An $S$-constraint is an application $R(\vec{x})$ of a $k$-ary relation $R \in S$ to a variable vector $\vec{x}=\left(x_{1}, \ldots, x_{k}\right)$ with $x_{i} \in X$ for all $i$. An $S$-formula is a conjunction of $S$-constraints where variables can be existentially quantified. In other words, an $S$-formula is a primitive positive formula of the type $\exists \vec{y} \bigwedge_{R \in S} R(\vec{x}, \vec{y})$. We also say that a relation is primitive positive definable from $S$ if it is the set of models of an $S$-formula. In the sequel, we use the graph $F^{\bullet}$ of functions $F$ for the set $S$. In this formalism, $F^{\bullet}$-constraints are written by means of equations of the type $f(x)=y$ for a function $f \in F$. Note that we can write an equation of the type $f(x)=g(y)$ for the expression $\exists z f(x)=z \wedge g(y)=z$ and $x=y$ for the expression $\exists z f(z)=x \wedge f(z)=y$. A constraint $R\left(x_{1}, \ldots, x_{k}\right)$ is satisfiable if there exists an interpretation $I: X \rightarrow D$ satisfying the membership condition $\left(I\left(x_{1}\right), \ldots, I\left(x_{k}\right)\right) \in R$. A conjunction $R_{1}(\vec{x}) \wedge \cdots \wedge R_{k}(\vec{x})$ is satisfiable if there exists an interpretation $I$ satisfying every constraint $R_{i}(\vec{x})$. An $S$-formula $\varphi(\vec{x})=\exists \vec{y} R_{1}(\vec{x}, \vec{y}) \wedge \cdots \wedge R_{k}(\vec{x}, \vec{y})$ is satisfiable if the conjunction $R_{1}(\vec{x}, \vec{y}) \wedge \cdots \wedge R_{k}(\vec{x}, \vec{y})$ is satisfiable. We write $I \models \varphi$ if the 
interpretation $I$ satisfies the formula $\varphi$. The set of models (or solutions) of a $k$ ary formula $\varphi$ is the relation $\operatorname{Sol}\left(\varphi\left(x_{1}, \ldots, x_{k}\right)\right)=\left\{\left(I\left(x_{1}\right), \ldots, I\left(x_{k}\right)\right) \mid I \models \varphi\right\}$. If the identity $\operatorname{Sol}(\varphi)=R$ holds then we say that the formula $\varphi$ implements the relation $R$.

Given a relation $R$ on a domain $D$ and $p$ an endomorphism of $R$, we denote by $p(R)$ the relation $\{p(t[1]), \ldots, p(t[k]) \mid t \in R\}$. Similarly for a set of relations $S$, we denote by $p(S)$ the set of relations $\{p(R) \mid R \in S\}$. The core of a constraint language $S$ is the subset $S_{c} \subseteq S$ such that every endomorphism on $S_{c}$ is an automorphism. Notice that if a constraint language $S$ is a core then every unary polymorphism $f$ of $S$ is bijective, i.e., $f$ is a permutation on the domain $D$. Observe that all cores of a constraint language $S$ are isomorphic. Thus, we write Cor $S$ to denote the unique core of $S$ up to renaming. Observe also that to compute a core of a constraint language $S$, the polymorphism $p \in \operatorname{Pol} S$ must be one of the unary polymorphisms of $S$ with the smallest range applied on each relation in $S$. Thus, the set $p(S)$ is a core of $S$.

A relational clone, also called a co-clone, is a set of relations closed under conjunction (Cartesian product), variable identification, and existential quantification (projection). The smallest co-clone containing a set of relations $S$, denoted by $\langle S\rangle$, is the set of relations primitive positive definable from $S$.

\section{Constraint Satisfaction Problems}

In general, a constraint satisfaction problem parametrized by a constraint language $S$, called a template, is defined as follows.

Problem: $\operatorname{CSP}(S)$

Input: An $S$-formula $\varphi\left(x_{1}, \ldots, x_{k}\right)$.

Question: Is $\varphi$ satisfiable?

In our context, a Co-Boolean Constraint Satisfaction Problem is a problem $\operatorname{CSP}\left(F^{\bullet}\right)$ for a set of co-Boolean functions $F$.

The following theorem allows us to use the algebraic approach for studying the complexity of co-Boolean CSPs. We introduce it in its general form, for two arbitrary sets of relations.

Theorem 1 (Jeavons [5]) Let $S_{1}$ and $S_{2}$ be sets of relations over D, with $S_{1}$ finite. If $S_{1} \subseteq\left\langle S_{2}\right\rangle$ holds then $\operatorname{CSP}\left(S_{1}\right)$ polynomial-time many-one reduces to $\operatorname{CSP}\left(S_{2}\right)$, denoted by $\operatorname{CSP}\left(S_{1}\right) \leq_{p} \operatorname{CSP}\left(S_{2}\right)$.

To study the complexity of co-Boolean constraint satisfaction problems $\operatorname{CSP}\left(F^{\bullet}\right)$ over a set of co-Boolean functions $F$, it is convenient to represent the set of graphs $F^{\bullet}$ in $H$-normal form.

Definition 2 ( $\boldsymbol{H}$-normal form) The $\boldsymbol{H}$-normal form of the of the set of functions $F=\left\{f_{1}, \ldots, f_{k}\right\}$ is the $(k+1)$-ary relation $F^{H}=\left\{\left(d, f_{1}(d), \ldots, f_{k}(d)\right) \mid\right.$ $d \in D\}$. The proper $H$-normal form is the right-hand side of $F^{H}$, namely $F_{r}^{H}=\left\{\left(f_{1}(d), \ldots, f_{k}(d)\right) \mid d \in D\right\}$. 
In other words, the $H$-normal form of a set of functions $F=\left\{f_{1}, \ldots, f_{k}\right\}$ is the $k+1$-ary relation $F^{H}$ which is the set of solutions of a $F^{\bullet}$-formula $\varphi\left(x, y_{1}, \ldots, y_{k}\right)$ defined by the conjunction $\bigwedge_{i \in\{1, \ldots, k\}} f_{i}(x)=y_{i}$ and the righthand side $F_{r}^{H}$ is the set of solutions of $\exists x \varphi\left(x, y_{1}, \ldots, y_{k}\right)$.

We will represent the relation $F^{H}$ in the form of a matrix, whose rows are the tuples of that relation. The columns of $F_{r}^{H}$ then represent the functions from $F$, except for the first column which is the enumeration of the domain $D$. When we speak about $F^{H}$, we call the first column the left-hand side and the other columns $F_{r}^{H}$ the right-hand side.

Proposition 3 The problems $\operatorname{CSP}\left(F^{\bullet}\right)$ and $\operatorname{CSP}\left(F^{H}\right)$ are polynomial-time equivalent.

Proof 1 By definition we have the inclusion $F^{H} \subseteq\left\langle F^{\bullet}\right\rangle$. Following Theorem 1 we have that $\operatorname{CSP}\left(F^{H}\right) \leq{ }_{p} \operatorname{CSP}\left(F^{\bullet}\right)$. To recover the graph $f_{i}^{\bullet}$ from $F^{H}$, existentially quantify all coordinates of $F^{H}\left(x_{0}, x_{1}, \ldots, x_{k}\right)$ except the coordinates 0 and $i$. Hence we get the inclusion $F^{\bullet} \subseteq\left\langle F^{H}\right\rangle$. This implies the reduction $\operatorname{CSP}\left(F^{\bullet}\right) \leq{ }_{p} \operatorname{CSP}\left(F^{H}\right)$, concluding the proof.

To classify the complexity of $\operatorname{CSP}\left(F^{\bullet}\right)$ for any set of unary functions $F$, it is enough to classify the complexity for $F$ containing all unary constant functions. This can be effectively done by means of cores. Recall that Cor $F^{\bullet}$ denotes the core of $F^{\bullet}$. Given a graph of a function $f^{\bullet}$ and an endomorphism $\pi$ on $f^{\bullet}$, $\pi\left(f^{\bullet}\right)$ denotes the relation $\left\{\left(\pi\left(d_{i}\right), \pi\left(d_{j}\right)\right) \mid\left(d_{i}, d_{j}\right) \in f^{\bullet}\right\}$. Similarly, given the graphs $F^{\bullet}, \pi\left(F^{\bullet}\right)$ denotes the set of relations $\left\{\pi\left(f^{\bullet}\right) \mid f^{\bullet} \in F^{\bullet}\right\}$. We need first to prove that a core of graphs is a set of graphs.

Lemma 4 Let $F$ be a set of functions. There exists a set of functions $G \subseteq F$ satisfying the equality $\operatorname{Cor} F^{\bullet}=G^{\bullet}$.

Proof 2 Assume that $F^{\bullet}$ is not a core, otherwise the claim is trivial. Take an unary polymorphism $p \in \operatorname{Pol} F^{\bullet}$ with a smallest range among all unary polymorphisms of $F^{\bullet}$. Then $p\left(F^{\bullet}\right)$ must be a core of $F^{\bullet}$, implying the inclusion Cor $F^{\bullet} \subseteq F^{\bullet}$, concluding the proof.

We also need to show that the complexity of our CSP problems do not change if we add all constant functions to the constraint language. Let $C_{s t}{ }_{D}$ denote the set of all unary constant functions over the domain $D$.

Lemma 5 For every set of functions $F$ on a domain $D^{\prime}$ there exists a set of functions $G$ on a domain $D \subseteq D^{\prime}$, such that $\operatorname{CSP}\left(F^{\bullet}\right)$ and $\operatorname{CSP}\left(G^{\bullet} \cup C s t_{D}^{\bullet}\right)$ are polynomial-time equivalent. More specifically, the set of functions $G$ satisfies the equality $G^{\bullet}=\operatorname{Cor} F^{\bullet}$ and $D$ is the domain of the constraint language $\operatorname{Cor} F^{\bullet}$.

Proof 3 The proof is a direct consequence of Theorems 4.4 and 4.7 in [1]. Theorem 4.4 shows that the problems $\operatorname{CSP}\left(F^{\bullet}\right)$ and $\operatorname{CSP}\left(\operatorname{Cor} F^{\bullet}\right)$ are polynomial-time equivalent. Theorem 4.7 shows that the problems $\operatorname{CSP}\left(\operatorname{Cor} F^{\bullet}\right)$ and $\operatorname{CSP}\left(\operatorname{Cor} F^{\bullet} \cup\right.$ Cst $\left._{D}^{\bullet}\right)$ are polynomial-time equivalent. 
According to the aforementioned Lemmas, we assume in the sequel that $F^{\bullet}$ is always a core for any set of functions $F$, and that $F$ contains all unary constant functions, in particular $\perp(x)=0$ and $\top(x)=1$ for all $x \in D$. Therefore the relation $F_{r}^{H}$ cannot be closed under any constant operation, since there exists no constant row in the matrix $F_{r}^{H}$.

\section{Dichotomy Theorem}

We present a complete characterization of complexity for co-Boolean constraint satisfaction problems. Recall that $F_{r}^{H}$ is a $|F|$-ary Boolean relation, also considered as a $|D| \times|F|$ Boolean matrix.

First, let us analyze the case when the Boolean relation $F_{r}^{H}$ is not closed under any of the four particular Boolean operations. This case behaves similarly to the case observed in [7].

Proposition 6 Let $F$ be a set of co-Boolean functions. If the relation $F_{r}^{H}$ is closed neither under majority, nor minority, nor conjunction, nor disjunction, then $\operatorname{CSP}\left(F^{\bullet}\right)$ is $\mathrm{NP}$-complete.

Proof 4 Recall that the relation $F_{r}^{H}$ can be implemented by the constraint $\exists x_{0} F^{H}\left(x_{0}, x_{1}, \ldots, x_{k}\right)$ where $|F|=k$, and remember we assume that $F$ contains the constant functions $\perp(x)=0$ and $\top(x)=1$ for all $x \in D$. Since the co-domain of each function from $F$ is $\{0,1\}$, the relation $F_{r}^{H}$ is Boolean. The Boolean relation $F_{r}^{H}$ cannot be 0 -valid or 1 -valid, since $F$ contains the constant functions $\perp$ and $\top$. Moreover, if the Boolean relation $F_{r}^{H}$ is not closed under majority, minority, conjunction, or disjunction, then according to Schaefer's result [7], $\operatorname{CSP}\left(F_{r}^{H}\right)$ is $\mathrm{NP}$-complete. Therefore by Proposition [3, $\operatorname{CSP}\left(F^{\bullet}\right)$ must be $\mathrm{NP}$-complete, too.

The cases left to be analyzed are those when $F_{r}^{H}$ is closed under majority, minority, conjunction, or disjunction. The first case is still compatible with Schaefer's results in [7.

Proposition 7 Let $F$ be a set of co-Boolean functions. If the Boolean relation $F_{r}^{H}$ is closed under majority or minority then $\operatorname{CSP}\left(F^{\bullet}\right)$ is in $\mathrm{P}$.

Proof 5 We only give the proof for majority, since the minority case is completely analogous. The left-hand side of $F^{H}$ represents the enumeration of the domain $D$ and therefore it can be seen as a numbering for the tuples in the Boolean relation $F_{r}^{H}$. If $F_{r}^{H}$ is closed under the majority operation, then major applied to any three (not necessarily distinct) tuples $a, b, c \in F_{r}^{H}$ results in a tuple $d \in F_{r}^{H}$, where major $(a, b, c)=d$. If we relate the tuples $a, b, c, d$ by their corresponding numberings $l(a), l(b), l(c), l(d)$ in the left-hand side of $F^{H}$, we get an extension of the majority operation on the whole domain $D$ in the following way. It is clear that the values $l(a), l(b), l(c)$ must be distinct for different rows of the matrix $F^{H}$. A majority operation major $(x, y, z)$ can assume an arbitrary value if the three values substituted for the variables $x, y, z$ are different. 
Hence we define major $(l(a), l(b), l(c))=l(d)$ if major $(a, b, c)=d$ for the tuples $a, b, c, d \in F_{r}^{H}$. This extension remains a majority operation.

The relation $F^{\bullet}$ is obviously closed under this extended majority operation. Jeavons proved in [6] that the closure of a constraint language $S$ under a majority operation implies the membership of $\operatorname{CSP}(S)$ in $\mathrm{P}$, therefore our result follows.

The second case differs from Schaefer's characterization [7]. Before introducing this case, let us remind that a semi-lattice operation is a binary associative, commutative and idempotent operation.

Proposition 8 Let $F$ be a set of co-Boolean functions. If $F_{r}^{H}$ is closed under conjunction or disjunction, and the first two tuples $(0, a)$ and $(1, b)$ of the relation $F^{H}$ satisfy the condition $a \preceq b$, where $\preceq$ is the coordinate-wise partial order on Boolean tuples induced by the order $0<1$, then $\operatorname{CSP}\left(F^{\bullet}\right)$ is in $\mathrm{P}$.

Proof 6 The proof is similar to that of Proposition 7 . We extend the operations of conjunction $\wedge$ or disjunction $\vee$ on $\{0,1\}$ from $F_{r}^{H}$ to semi-lattice operations of $F^{H}$ by using the induced numbering of tuples from the first coordinate of $F^{H}$. Jeavons [6] proved that the closure of a constraint language $S$ under a semilattice operation implies the membership of $\operatorname{CSP}(S)$ in $\mathrm{P}$. Then $\operatorname{CSP}\left(F^{H}\right)$ is in $\mathrm{P}$ and therefore also $\operatorname{CSP}\left(F^{\bullet}\right)$ by Proposition 3 .

Finally we show that the remaining cases, when the two first tuples in $F^{H}$ are not ordered compatibly with their position, are NP-complete.

Proposition 9 Let $F$ be a set of co-Boolean functions. If $F_{r}^{H}$ is closed under conjunction or disjunction, but neither under majority nor minority, and the first two tuples $(0, a)$ and $(1, b)$ from $F^{H}$ do not satisfy the condition $a \preceq b$, with $\preceq$ being the coordinate-wise partial order on Boolean tuples induced by the order $0<1$, then $\operatorname{CSP}\left(F^{\bullet}\right)$ is $\mathrm{NP}$-complete.

Proof 7 Note that $F_{r}^{H}$ cannot be closed under both conjunction and disjunction since this would imply that it is closed under majority. It follows from the identity major $(x, y, z)=(x \vee y) \wedge(y \vee z) \wedge(z \vee x)$.

Consider the first two tuples in $F^{H}$, namely $(0, a)$ and $(1, b)$. The condition $a \preceq b$ is falsified, therefore there must exist a coordinate $i$ with $a[i]=1$ and $b[i]=0$. Hence there exists a function $f \in F$ with $f(0)=1$ and $f(1)=0$. Hence we have $\{01,10\} \subseteq f^{\bullet}$. The relation $\{01,10\}$ is closed neither under conjunction, nor under disjunction. Therefore also the relation $f^{\bullet}$ cannot be closed under minimum or maximum, hence also the relation $F^{H}$ cannot be closed under these two operations either. The Boolean relation $F_{r}^{H}$ is by assumption closed neither under majority nor under minority. Hence $\operatorname{CSP}\left(F_{r}^{H} \cup f^{\bullet}\right)$ is NPcomplete according to Schaefer [7]. Since the inclusion $F_{r}^{H} \cup f^{\bullet} \subseteq\left\langle F^{H}\right\rangle$ holds, by Theorem 1 and Proposition 3 we have $\operatorname{CSP}\left(F^{\bullet}\right) \mathrm{NP}$-complete.

By regrouping the proposition of this section, we derive the following complete classification of complexity for homogeneous co-Boolean CSPs. 
Theorem 10 (Dichotomy Theorem) Let $F$ be a set of homogeneous co-Boolean functions and $G^{\bullet}$ be a set a relations such that $G^{\bullet}=\operatorname{Cor} F^{\bullet}$ holds. If the Boolean relation $G_{r}^{H}$ is closed under majority or minority, or if it is closed under conjunction or disjunction and the two first tuples $(0, a),(1, b)$ from the matrix $G^{H}$ satisfy the condition $a \preceq b$, then $\operatorname{CSP}\left(F^{\bullet}\right)$ is in $\mathrm{P}$. Otherwise $\operatorname{CSP}\left(F^{\bullet}\right)$ is NP-complete.

Proof 8 This follows from Lemmata 4 and $[5$, and Propositions 6 to 9 .

\section{Concluding Remarks}

The result presented in this short note is a first step toward the study of the complexity of CSP over unary functions. We have proved a Dichotomy Theorem for the complexity of homogeneous co-Boolean constraint satisfaction problems for every finite domain $D$. Even if this Dichotomy Theorem is mainly based on the famous Schaefer's theorem, it presents a first study of the new angle of attack proposed by Feder et al., and allow us to easily determinate the complexity of homogeneous co-Boolean CSP thanks to the $H$-normal form, which was not trivial so far.

A natural extension of this work would be the study of the complexity of $\operatorname{CSP}\left(F^{\bullet}\right)$ where $F$ is a set of unary functions not necessary sharing anymore the same co-domain $\{0,1\}$, i.e. non-homogeneous co-Boolean functions where co-domains of functions $f \in F$ are independent. It would be also interesting to study the complexity of co-ternary CSP, where the constraint language is built upon unary functions sharing the same co-domain $\{0,1,2\} \subseteq D$. Similarly to our dichotomy theorem, which differs from Schaefer's Dichotomy Theorem in [7, we conjecture that a dichotomy theorem for the latter problem will also be different from Bulatov's Dichotomy Theorem in [2].

\section{Acknowledgments}

The author would like to thank Miki Hermann and Gustav Nordh for theirs helpful advices and ideas, allowing him to start this study.

\section{References}

[1] A. Bulatov, P. Jeavons, and A. Krokhin. Classifying the complexity of constraints using finite algebras. SIAM Journal on Computing, 34(3):720$742,2005$.

[2] A. A. Bulatov. A dichotomy theorem for constraint satisfaction problems on a 3-element set. Journal of the Association for Computing Machinery, 53(1):66-120, 2006. 
[3] T. Feder, F. Madelaine, and I. Stewart. Dichotomies for classes of homomorphism problems involving unary functions. Theoretical Computer Science, 314(1-2):1-43, 2004.

[4] T. Feder and M. Y. Vardi. The computational structure of monotone monadic SNP and constraint satisfaction: a study through Datalog and group theory. SIAM Journal on Computing, 28(1):57-104, 1998.

[5] P. Jeavons. On the algebraic structure of combinatorial problems. Theoretical Computer Science, 200(1-2):185-204, 1998.

[6] P. Jeavons, D. Cohen, and M. Gyssens. Closure properties of constraints. Journal of the Association for Computing Machinery, 44(4):527-548, 1997.

[7] T. J. Schaefer. The complexity of satisfiability problems. In Proceedings 10th Symposium on Theory of Computing (STOC'r8), San Diego (California, USA), pages 216-226, 1978. 